\title{
Optimal Predictive analytics of Pima Diabetics using Deep Learning
}

\author{
Sushant Ramesh, H. Balaji ${ }^{1}$, N.Ch.S.N Iyengar ${ }^{*}$ and Ronnie D. Caytiles ${ }^{2}$ \\ SCOPE, VIT University, Vellore-632014 \\ ${ }^{1}$ Sreenidhi Institute of Science and Technology, Ghatkesar, Hyderabad, India \\ ${ }^{2}$ Multimedia Engineering Department, Hannam University, Daejeon, Korea \\ sushant.ramesh2013@vit.ac.in,rdcaytiles@gmail.com, \\ srimannarayanach@sreenidhi.edu.in
}

\begin{abstract}
An intelligent predictive model using deep learning is proposed to predict the patient risk factor and severity of diabetics using conditional data set. The model involves deep learning in the form of a deep neural network which helps to apply predictive analytics on the diabetes data set to obtain optimal results. The existing predictive models is used to predict the severity and the risk factor of the diabetics based on the data which is processed. In our case Firstly, a feature selection algorithm is run for the selection process. Secondly, the deep learning model has a deep neural network which employs a Restricted Boltzmann Machine (RBM) as a basic unit to analyse the data by assigning weights to the each branch of the neural network. This deep neural network, coded on python, will help to obtain numeric results on the severity and the risk factor of the diabetics in the data set. At the end, a comparative study is done between the implementation of this model on type 1 diabetes mellitus, Pima Indians diabetes and the Rough set theory model. The results add value to additional reports because the number of studies done on diabetes using a deep learning model is few to none. This will help to predict diabetes with much more precision as shown by the results obtained.
\end{abstract}

Keywords: Deep Learning, Diabetes Mellitus, Restricted Boltzmann Machine

\section{Introduction}

Diabetes mellitus, commonly known as diabetes, is group of metabolic diseases which marks high blood sugar levels over a prolonged period. As per the survey done by International Diabetes Federation, in 2015, it was estimated that 415 million individuals are affected by diabetes around the world. And the number is estimated to rise to 642 million individuals by the year 2040. Type 1 diabetes is a disorder in which the pancreas can no longer produce insulin. It is also called juvenile diabetes primarily because of its presence in both children and adults. However, statistics show that only 5\% of all the type 1 cases are diagnosed in adulthood. It is sometimes referred to as insulin-dependent diabetes mellitus and has no cure. If one has it, they must take insulin to survive. According to the WHO (World Health Organization) the number of children having type 1 diabetes is very high as mentioned in the motivation. Hence it can be said that Diabetes is a serious chronic disease. Doctors usually take patients' blood samples and check the sugar concentration in their blood in order to diagnose them with diabetes. This is a highly time-consuming process and there are many other features which need to be reviewed while attempting to detect whether a patient is diabetic or not. These other factors are: insulin, body mass index, blood pressure and age. Even family history can play a vital role in a person's diabetic status. If a patient's ancestors show a presence of

Received (July 18, 2017), Review Result (September 1, 2017), Accepted (September 15, 2017) 
diabetes, then there is a high chance of the patient exhibiting symptoms of diabetes as well. Presently, there is no non-intrusive technique to detect if a person has type 1 diabetes and how severe the diabetes will affect him/her. Hence the need arises for some sort of efficient application to predict the onset of type 1 diabetes in patients for a quicker diagnosis which can lead to quicker treatment. The prevalence of diabetes varies among tribes, bands, pueblos, and villages, and ranges from less than 5\%, to 50\% for diagnosed diabetes. There are more than 550 federally recognized tribes, bands, pueblos, and villages in the United States.Pima Indians, on the average, are twice more likely to die from complications of diabetes than non-Pima Indians. They are the fattest population group in the United States, and they have the maximum occurrence of diabetes in the world. Despite unprecedented research by the National Institute of Arthritis, Diabetes and Digestive and Kidney Diseases (NIH) and good care by the Indian Health Service (IHS), these issues have only since they were first documented in the 1960s. There are multiple causes leading to the incredibly high occurrence of diabetes: environmental, genetic, cultural, and psychosocial. The presence of type 2 diabetes which is non-insulindependent diabetes is more in Pima Indians than it is in any other geographically defined population on earth. While people diagnosed with type 1 diabetes can be easily treated with insulin shots, type 2 diabetes is much more difficult to remedy. Patients diagnosed with type 2 diabetes i.e., non-insulin dependent diabetes must be made to follow a special diet and may moreover, take diabetes pills. At times, the need may arise for them to be administered with insulin shots. The best solution to treat type 2 diabetes in Pima Indians is to diagnose the onset of diabetes in the early stages of life, primarily childhood. Deep learning involves working on a model quite similar to that of the human brain. It has the capability to decode complex problems much like that of the human brain. Deep neural networks can handle massive sets of data (as done in this project), grasp complex tasks and handle data with small manual inputs. The basic concept behind deep learning is that it produces a rendering of raw data at a higher level. It is a learning method with a deep architecture and algorithms which can learn features that have no labels i.e., unsupervised data. This drives the idea that a heavy amount of unsupervised learning is required. The ability of deep neural networks to follow the same decision process as that of the human brain, along with the methodical learning algorithms that can ensure this ability, make it fast in terms of processing speed and it can also handle data of high dimensions better than simple machine learning algorithms. RStudio is an IDE for $\mathrm{R}$ programming. It includes an editor which highlights the syntax and supports direct code execution, as well as tools and functions for plotting graphs, checking history, debugging programs and workspace management. RStudio was written in $\mathrm{C}++$ and uses the QT framework for its GUI for functions such as plotting of graphs and charts.

The following are the advantages offered by RStudio:

- Integrated support for Git and Subversion

- Supports authoring HTML, PDF, Word Documents, Slide Shows.

- Supports interactive graphics with Shiny and ggvis.

- Integrates tools used in R into a single environment.

TensorFlow is an open source software library for machine learning across a range of tasks, and developed by Google to meet their needs for systems capable of building and training neural networks to detect and decipher patterns and correlations, analogous to the learning and reasoning which humans use. It is currently used for both research and production at Google products, often replacing the role of its closed-source predecessor, DistBelief. It offers the following advantages:

- Speed: TensorFlow 1.0 is highly fast. XLA lays the groundwork for even more performance improvements in the future.

- Flexibility: TensorFlow 1.0 introduces a high-level API for TensorFlow, with tf.layers, tf.metrics, and tf.losses modules. A new tf.keras module is also 
included, that provides full compatibility with Keras, another popular highlevel neural networks library.

- Production-readiness: TensorFlow 1.0 provides Python API stability, making it easier to pick up new features without worrying about breaking one's existing code.

\section{Literature Survey/Related Work}

[1] AsmaShaheen Khan, Waqas Ahmed proposed an Intelligent decision support system in diabetic ehealth care from the perspective of elders. The proposed system stores the patients' information and gives them optimal advices according to their condition entered by them. It also provides adequate and detail information about the patient to the health-care providers that help them to take an optimal decision about the patients. In [2] Tawfik Saeed Zeki, Mohammad V. Malakooti, Yousef Ataeipoor, TalayehTabibi proposed an expert system for diabetes diagnosis. After data acquisition and designing a rule-based expert system, this system has been coded with VP_Expert Shell and tested in ShahidHasheminezhad Teaching Hospital affiliated to Tehran University of Medical Sciences and final expert system was presented which could diagnose all kinds of diabetes. In [3] Rahman Ali, Jamil Hussain, Muhammad Hameed Siddiqi, Maqbool Hussain and Sungyoung Lee, proposed a Hybrid Rough Set Reasoning Model for Prediction and Management of Diabetes Mellitus. When a new incoming data is evaluated and compared against the knowledge base derived from the rough classification to classify the type of diabetes of the patient. In [6] T.P. Kamble and Dr. S.T. Patil proposed a system where Deep learning based Restricted Boltzmann machine approach is used to detect whether patient is diabetic or not as Restricted Boltzmann machine is popular for classification and recognition purpose. To detect either patient is having type 1 or type 2 diabetes decision tree technique used. In [8] Riccardo Miotto, Li Li, Brian A. Kidd, Joel T. Dudley presented a novel unsupervised deep feature learning method to derive a general-purpose patient representation from EHR data that facilitates clinical predictive modelling. Evaluations using 76,214 test patients comprising 78 diseases from diverse clinical domains and temporal windows were performed. The findings indicate that deep learning applied to EHRs can derive patient representations that offer improved clinical predictions, and could provide a machine learning framework for augmenting clinical decision systems. In [12] Margret Anouncia S., Clara Madonna L. J., Jeevitha P., Nandhini R. T. proposed a design for a Diabetic Diagnosis System using Rough Sets where the authors created a knowledge base from the existing data- set using upper and lower approximations and when a new incoming data is collected and evaluated to compute the equivalence classes. These equivalence are then compared against the knowledge, the system helped the user discern the type of diabetes. In [13] RahmatZolfaghari proposed a three-layer hierarchy multi- classifier. And min max normalization is applied on the data to avoid the numerical difficulties in calculations. First Layer detects the features then next layer separately classify using back propagation and SVM (Support Vector Machine) then in next layer combine output from both layers the ensemble means combination or fusion of 2 or more algorithms. In [14] K. Sridar, Dr. D. Shanthi used the back propagation and Apriori algorithm for the diabetes detection purpose. First the input is taken from the user. The input from user is glucose level which was detected in blood sample then back propagation algorithm is applied on the inputs then the output of back propagation is applied as input to association rule mining. The final output shows the percentage of diabetes in the patient. In [15] Gaganjot Kaur, Amit Chhabra proposed classification which used Decision tree algorithm to predict class whether patient is diabetic or not. The labelled data is feed as input. The leaf of the tree $\mathrm{j} 48$ acts as the class labels. The information gain is calculated for each attribute. Then the gain in information is calculated that would result from a test on the attribute. In [21] Jack 
W. Smith, J.E. Everhart, W.C. Dickson, W.C. Knowler, R.S. Johannes tested the ability of an early neural network model, ADAP, to forecast the onset of diabetes mellitus in a high risk population of Pima Indians. The algorithm's performance was analyzed using standard measures for clinical tests: sensitivity, specificity, and a receiver operating characteristic curve. They further examined these methods by comparing the ADAP results with those obtained from logistic regression and linear perceptron models using precisely the same training and forecasting sets.

\section{Gaps Identified in the Survey}

1) Limitations of Perceptron learning: The lack of the ability to handle any other values other than input and output made it very inflexible. In order to handle linearly non separable functions such as XOR and XNOR, more layers needed to be added. 2) Limitations of back propagation are local minima and network paralysis.

\section{Local minima-}

The network keeps changing its weights. Due to this, the error is reproduced and the algorithm gets stuck. Thus, error will not reduce further.

\section{Network paralysis-}

The weights are kept to very large values the large values force most of the units of network to operate in extreme values in such region derivation of activation functions is very small. The number of times back propagation requires the input pattern presentation for which weights need to update so that network will settle down to its optimal solutions 3) Limitations of SVM:

- Performance of the SVM is primarily dependent on the kernel function that has been selected

- SVM speed is a lot lesser during the training and testing phases.

4) As the reducts generation problem is an NP-Hard problem, we need to implement the LEM2 algorithm for extraction of rules. 5) It is not possible to determine whether Rough clustering is a better alternative or rough classification is better because no proper comparative study has been conducted.

\section{Motivation}

Type 1 diabetes mellitus (T1DM) itself comprises of roughly around $10 \%$ of the reported cases with diabetes. T1DM usually affects children where the causes are of the disease is unknown and no known ways to prevent the T1DM. The study [9] performed and published by Jane L. Chiang et al., estimated about 80000 children showing symptoms and developing the disease each year. The prevalence of Type 2 diabetes in Native American communities has increased dramatically during the second half of this century. Although many factors contribute to this marked increase, studies indicate that a trend away from traditional lifestyles in favor of westernization, with accompanying increases in body weight and diminished physical activity, is largely to blame. Complications from diabetes are major causes of death and health problems in most Native American populations. Of equal concern is the fact that Type 2, or adult-onset diabetes, is increasingly being discovered in Native American youth. Diabetes rates are highest in full-blooded Native Americans, as first observed in Choctaw Indians in 1965 and subsequently in other tribes. The prevalence of diabetes in residents of the Pima community in Arizona is the highest in individuals of full Native American heritage. About 50 percent of the tribe between the ages of 30 and 64 have diabetes. Prevalence of 
Type 2 diabetes among all Native American tribes in the United States is 12.2 percent of those over 19 years of age.

The serious complications of diabetes are increasing in frequency among Native Americans. Of major concern are increasing rates of kidney failure, amputations, and blindness. Among people with diabetes, the rate of diabetic end-stage renal disease is six times higher among Native Americans. Diabetes is the most frequent cause of nontraumatic lower limb amputations. Amputation rates among Native Americans are 3-4 times higher than the general population. Diabetic retinopathy is a term used for all abnormalities of the small blood vessels of the retina caused by diabetes, such as weakening of blood vessel walls or leakage from blood vessels. Diabetic retinopathy occurs in 18 percent of Pima Indians and 24.4 percent of Oklahoma Indians. The motivation behind pursuing a working model in the field of diabetes, in general, is to guide the doctors and the guardians of the affected children to catch the disease in its nascent stage and based on the severity of diabetes, accordingly provide apt treatment to the affected children.

\section{Framework}

The deep learning framework used in this project is TensorFlow. While the reference implementation runs on single devices, TensorFlow can run on multiple CPUs and GPUs (with optional CUDA extensions for general-purpose computing on graphics processing units). TensorFlow is available on 64-bit Linux, macOS, and mobile computing platforms including Android and iOS.

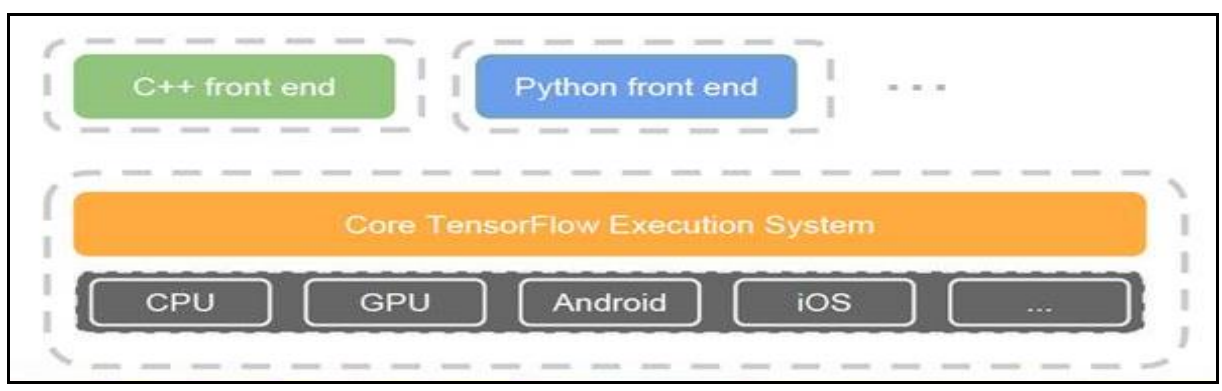

Figure 1. Deep Learning Framework - TensorFlow

The deep learning model chosen for this endeavour is a Recurrent Deep Neural Network (RNN). The most widely used neural network is a feed-forward neural network (MLP). However, that has not been chosen for this project specifically because even when both neural networks are well trained, a RNN uses more information than a MLP. Also, while a MLP can approximate any function to an arbitrary precision, the accuracy obtained by a RNN is much higher due to the presence of a layer that considers inputs from different time points i.e. the recurrent formation of the neural network.

\section{Methodology Adopted}

Wherever The data sets considered for this project were obtained from the online Machine Learning UCI repository. The links of the data sets are given. T1DM [4]: https://archive.ics.uci.edu/ml/datasets/diabetesPima Indians Diabetes [5]: https://archive.ics.uci.edu/ml/datasets/pima+indians+diabetes. The proposed system follows the steps as shown in Figure 2, which is the process flow diagram. 


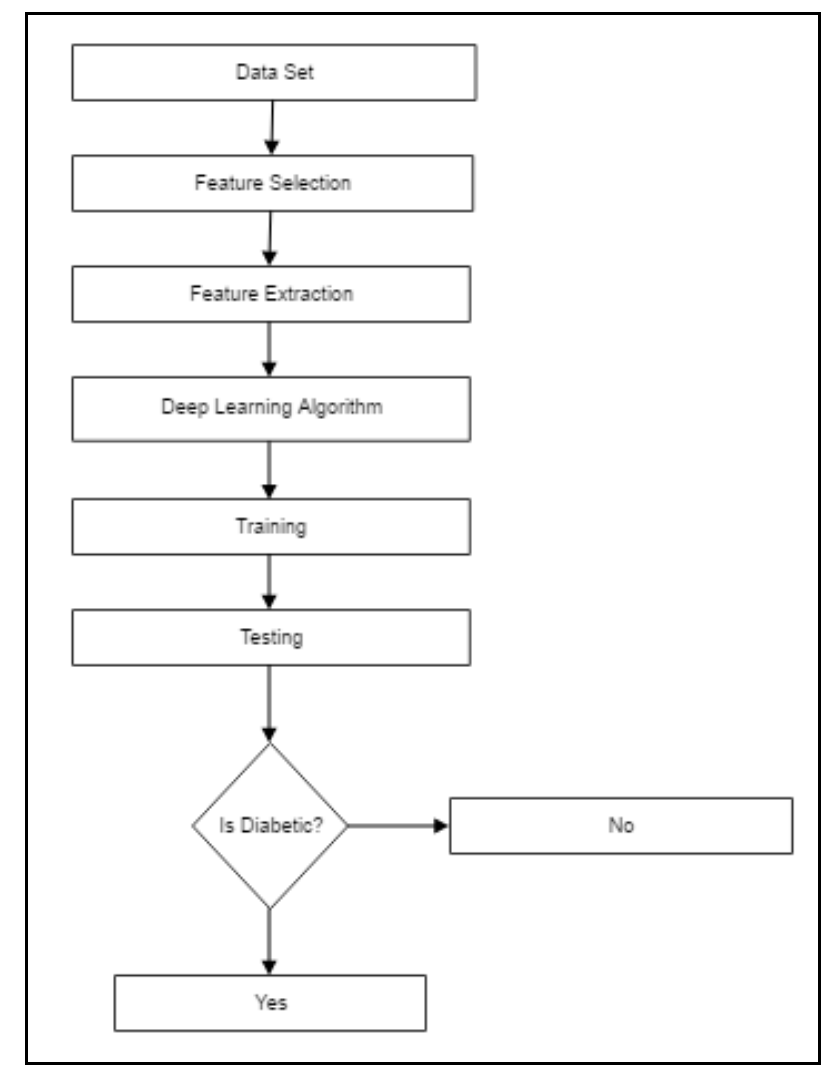

\section{Figure 2. Process Flow Diagram for the Deep Learning Model}

First and foremost, the feature is selected based on the weights provided to the different symptoms in the deep neural network. Those features are then extracted and their data values are applied to the Restricted Boltzmann machine for classification. Restricted Boltzmann machine is model which is having structure like bipartite graph (i.e., there is no intra-layer communication between the nodes of the same layer of the neural network) and it is an energy based model. The aforementioned classifier detects whether the candidate is diabetic or not. In the data pre-processing step, the features are selected from the file on the basis of their uniqueness. The attributes, in descending order of their importance are Glucose, BMI, Age, Pregnancies, Diabetes Pedigree Function, Blood Pressure, Skin Thickness and Insulin. The graphical representation of this order as calculated on RStudio using the Random Forest Algorithm is presented in Figures 3 and 4. The Random Forest Package in $\mathrm{R}$ includes the following functions which have been used (in the same order as they have been defined):

1) classCenter() - Prototypes of groups.

2) grow () - Add trees to an ensemble

3) getTree() - Extract a single tree from a forest.

4) Importance() - Extract variable importance measure

5) Margin() - Margins of randomForest Classifier

6) outlier() - Compute outlying measures

7) plot.randomForest() - Plot method for randomForest objects

The plot.randomForest() function was used on all the objects i.e., attributes for the diabetes data sets. Hence, a graphical representation of the attributes was obtained according to their uniqueness in the data sets, thus affecting their influence in the onset of diabetes. 
Type 1 Diabetes Mellitus

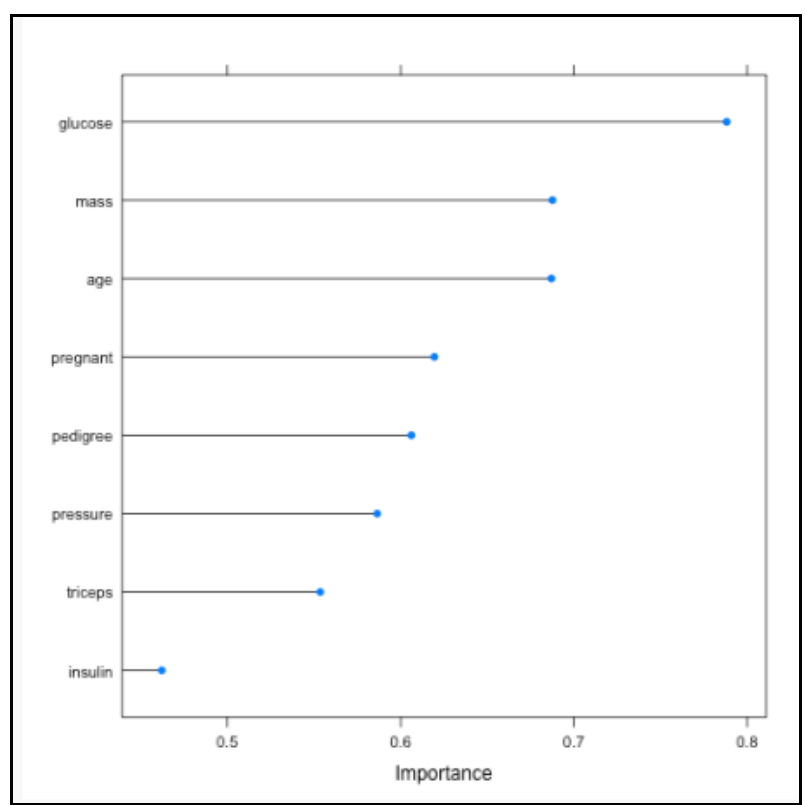

Figure 3. Ranking the Attributes for Feature Selection

Table 1. Attributes Arranged by Priority with Assigned Weights

\begin{tabular}{|l|l|}
\hline Attribute & Assigned Weight \\
\hline Glucose & 0.8 \\
\hline BMI & 0.7 \\
\hline Age & 0.7 \\
\hline Pregnancies & 0.615 \\
\hline Diabetes Pedigree Function & 0.61 \\
\hline Blood Pressure & 0.59 \\
\hline Skin Thickness & 0.55 \\
\hline Insulin & 0.47 \\
\hline
\end{tabular}


Pima Indians Diabetes

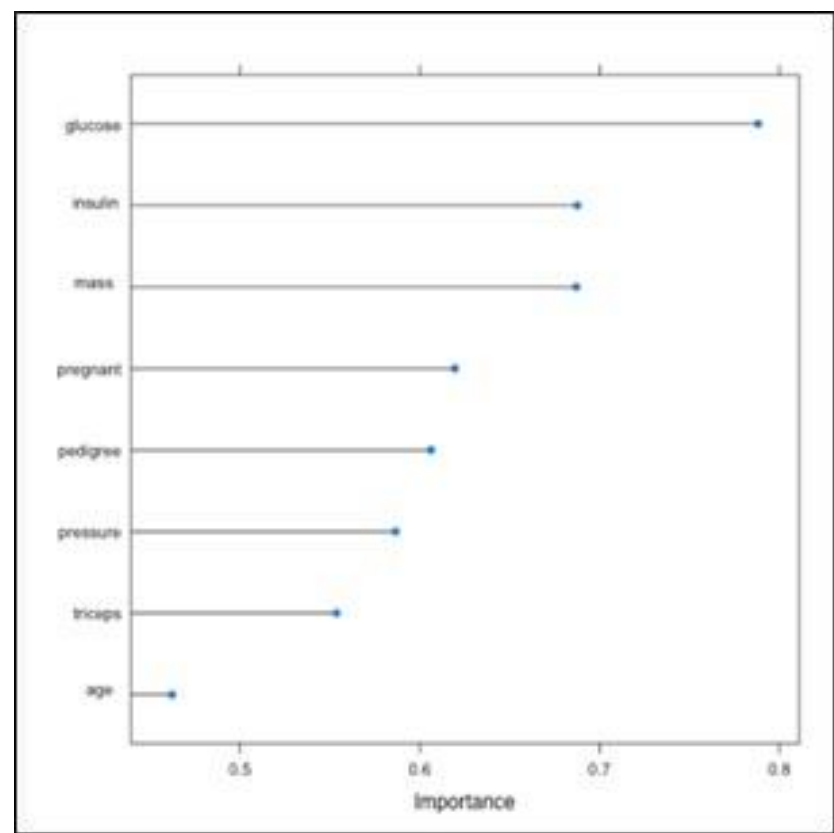

Figure 4. Ranking the Attributes for Feature Selection in Pima Indians Diabetes

Table 2. Attributes Arranged by Priority with Assigned Weights for Pima Indians Diabetes

\begin{tabular}{|l|l|}
\hline Attribute & Assigned Weight \\
\hline Glucose & 0.8 \\
\hline Insulin & 0.7 \\
\hline BMI & 0.7 \\
\hline Pregnancies & 0.615 \\
\hline Diabetes Pedigree Function & 0.61 \\
\hline Blood Pressure & 0.59 \\
\hline Skin Thickness & 0.55 \\
\hline Age & 0.47 \\
\hline
\end{tabular}

Next, the data set is normalized with respect to these features through min-max normalization in order to get an input vector range between 0 and 1 , and to avoid computation complexity. And next the dataset is divided into training dataset and test dataset such as $80 \%$ of training dataset and $20 \%$ of test dataset. Upper bound (UB) $=1$ and lower bound $(\mathrm{LB})=0$. Equation (1) as shown below is used to find the min-max normalization of data. 


$$
X_{N o r}=\frac{\left(X-X_{M i n}\right)}{\left(X_{M a x}-X_{M i n}\right)}(U B-L B)
$$

The graphical structure of a Restricted Boltzmann Machine (RBM) has an undirected graph. It has a visible layer and a hidden layer. The input is directly clamped with the visible layer. It has a layer of visible units connected to a layer of hidden units but no connections within a layer. Typically, RBMs use binary units for both visible and hidden variables. But the real valued data can be applied to RBM which is having structure as Gaussian Bernoulli type architecture. To model real-valued data, a modified RBM with binary logistic hidden units and real-valued Gaussian visible units can be used.

A Restricted Boltzmann machine is a particular type of a Markov random field which has two layers. One layer of an RBM consists of visible input units, $v$ is ranging from 0 to 1 , which are connected to the other layer of hidden stochastic units is $\mathrm{h}$ which is either 0 or 1. Figures 5 and 6 show the basic structure of an RBM. The distribution of state $\{v, h\}$ of an RBM is specified by the energy function ${ }^{[16]}$ as shown in equation (2).

$$
E(v, h)=\sum_{j} \frac{\left(v_{i}-b_{i}\right)^{2}}{\sigma^{2}}-\left(\sum_{i, j} \frac{v_{i}}{\sigma_{i}} \times w_{i, j} \times h_{j}\right)-\sum_{j} c_{j} h_{j}
$$

In a Restricted Boltzmann Machine, the visible units are used to find the distribution of the hidden units while the hidden units are used to compute the distribution of visible unit until the stable state is obtained. Markov chain is important in RBM as it helps to get samples from probability distribution such as Gibbs' distribution.

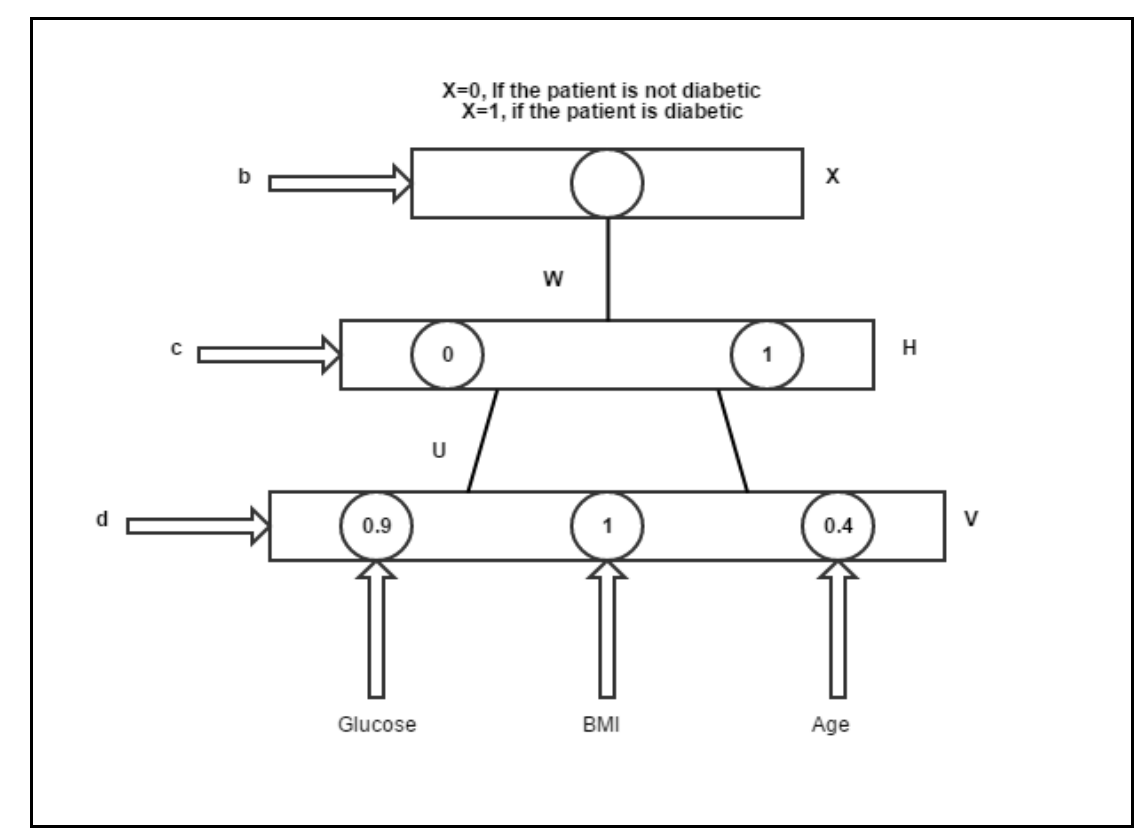

Figure 5. T1DM Architecture for Restricted Boltzmann Machine for Classification based on the 3 top Priority Attributes 


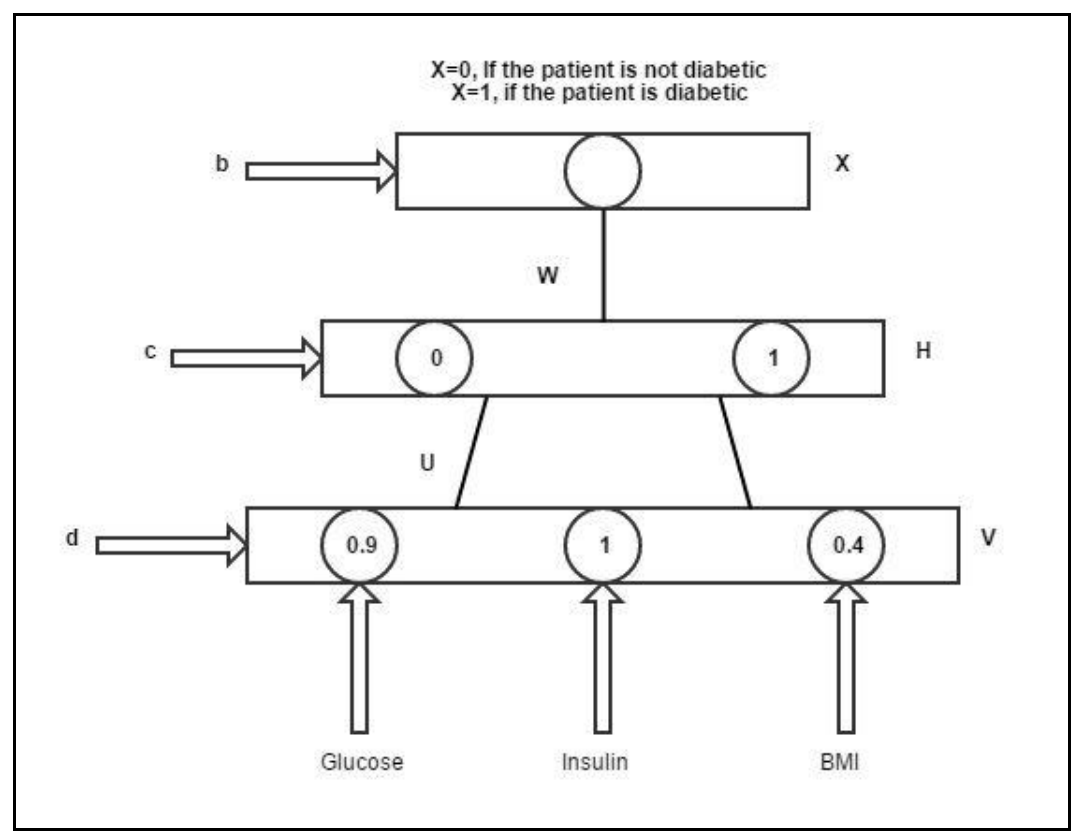

\section{Figure 6. Pima Indians Diabetes Architecture for Restricted Boltzmann Machine for Classification based on the 3 Top Priority Attributes}

In Figures 5 and 6, $\mathrm{H}$ depicts the hidden layers of the deep neural network while $\mathrm{V}$ depicts the visible layer of the same neural network. In the visible layer, all the attributes are taken directly from the data set. In the hidden layer, the computation takes place using equations (1) and (2).

Gibbs sampling (As shown in Figure 7):

1) Updation starts at a random state of the visible unit

2) All the hidden units must then be updated in parallel

3) Updation and construction of the visible unit is also done in parallel

4) Repeat steps 1 to 3 for the entire training example

\section{Algorithm for Gibbs' Sampler}

1. Initialize $\mathrm{x}(0) \sim \mathrm{q}(\mathrm{x})$

2. for iteration $i=1,2, \ldots$ do

2.1. $x^{(i)} \sim p\left(X_{D}=x_{D} \mid X_{1}=x^{(i)}{ }_{1}, X_{2}=x^{(i)}{ }_{2}, \ldots, X_{D}=x^{(i)}{ }_{D-1}\right)$

end for

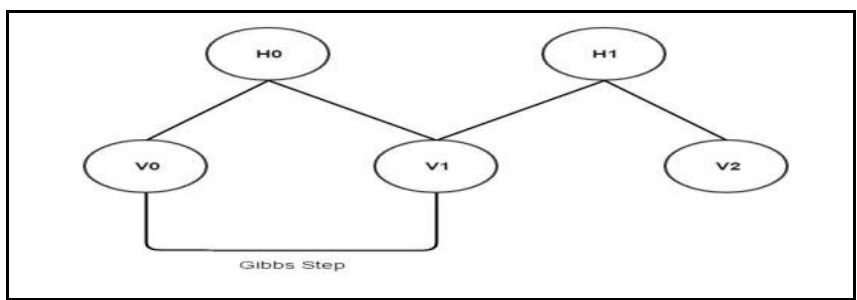

Figure 7. Gibbs Sampling Step 


\section{Need for Gibbs Sampling:}

The Gibbs sampler algorithm provides a solution to one very important problem which is sampling values from a probability distribution. As shown in the algorithm above, the Gibbs sampler provides a method to efficiently approximate a joint distribution under one condition: we should easily be able to sample from the conditional distribution of each $X_{i}$.

\section{Experimental Setup and Simulation Results}

Type Figure 8 as shown below is a list of all the features/parameters present in the data set obtained from the UCI Machine Learning Repository. Furthermore, a small snippet of the deep learning code is also on display.

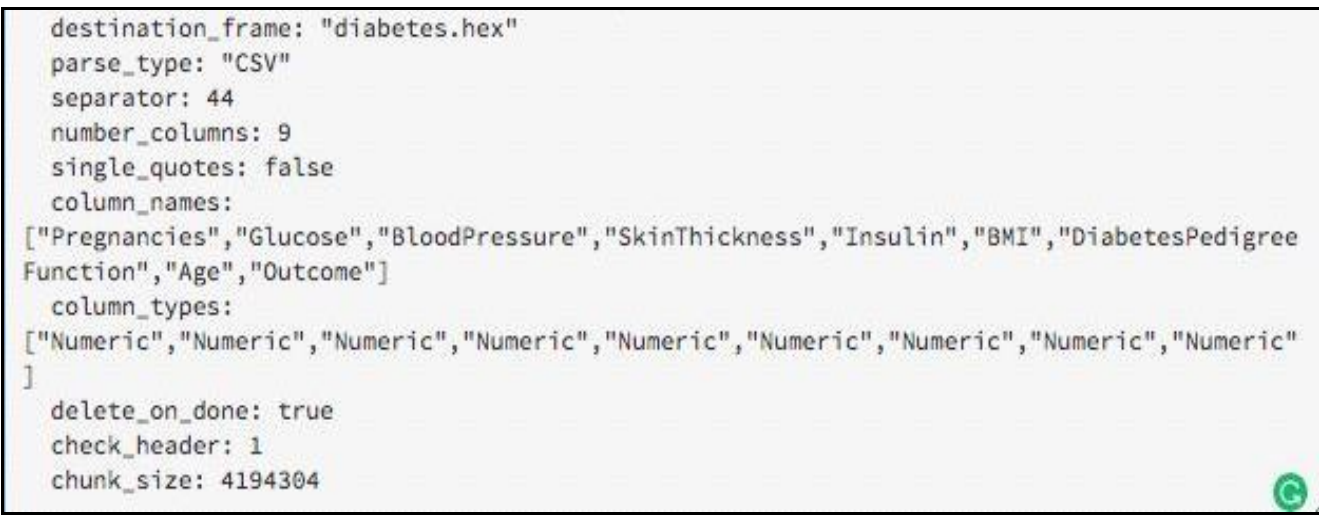

\section{Figure 8. Attributes of both the Diabetes Data Sets}

The deep neural network was trained and tested in TensorFlow as shown in Figure 9. On constructing and training a deep neural network i.e., an RBM, and simulating the data sets $[4,5]$, the following results were obtained:

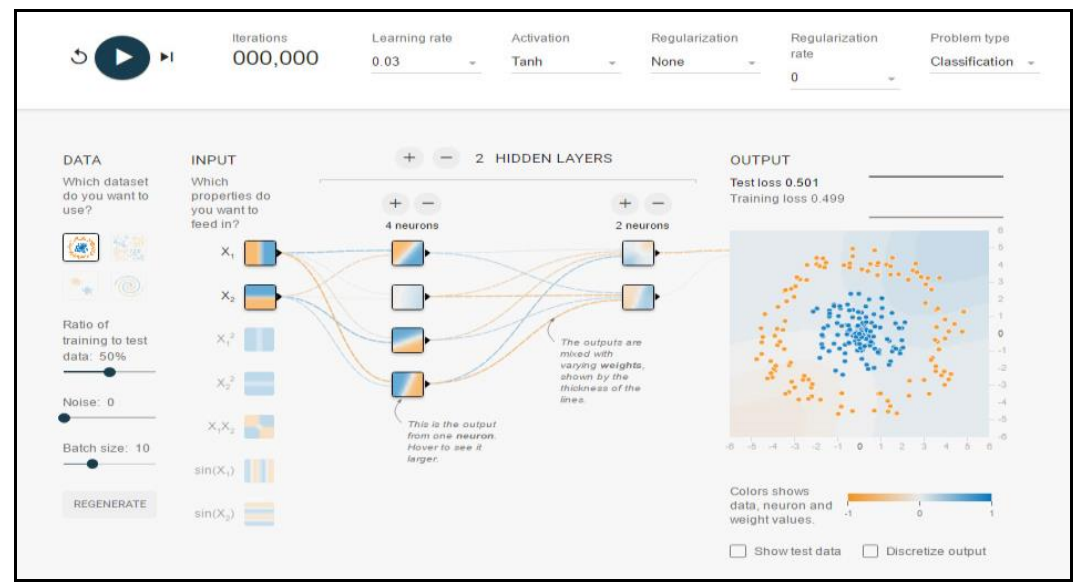

Figure 9. Physical Representation of the Deep Neural Network

\section{RESULTS FOR TYPE 1 DIABETES MELLITUS:}

Initial Result obtained after applying logistic regression on data generated from restricted Boltzmann machine:-

Correctly Classified Instances: $117 \quad$ (78\%)

Incorrectly Classified Instances: $33 \quad$ (22\%)

Kappa statistic: $\quad 0.5175$ 
Mean absolute error: $\quad 0.3006$

Root mean squared error: $\quad 0.398$

Relative absolute error: $\quad 83.0092 \%$

Root relative squared error: $\quad 92.7824 \%$

Coverage of cases (0.95 level): $\quad 96.05 \%$

Mean rel. region size (0.95 level): $86.05 \%$

Total Number of Instances: $\quad 150$

Table 3. Showing the Correctly and Incorrectly Classified Instances using RBM

\begin{tabular}{|c|c|c|c|}
\hline & \multicolumn{3}{|c|}{$\begin{array}{l}\text { Predicted } \\
\text { Classes }\end{array}$} \\
\hline \multirow{3}{*}{$\begin{array}{l}\text { Actual } \\
\text { Classes }\end{array}$} & & 0 & 1 \\
\hline & 0 & $1^{9}$ & 9 \\
\hline & $\mathbf{1}$ & $4^{2}$ & $6^{2}$ \\
\hline
\end{tabular}

\section{RESULTS FOR PIMA INDIANS DIABETES:}

Initial Result obtained after applying logistic regression on data generated from restricted Boltzmann machine:-

Correctly Classified Instances: $622 \quad(81 \%)$

Incorrectly Classified Instances: $146 \quad$ (19\%)

Kappa statistic: $\quad 0.5297$

Mean absolute error: $\quad 0.211$

Root mean squared error: $\quad 0.304$

Relative absolute error: $\quad 80.1856 \%$

Root relative squared error: $\quad 91.4408 \%$

Coverage of cases ( 0.95 level): $\quad 97.15 \%$

Mean rel. region size (0.95 level): $89.35 \%$

Total Number of Instances: $\quad 768$

Table 4. Showing the Correctly and Incorrectly Classified Instances using RBM

\begin{tabular}{|l|l|l|l|}
\hline \multirow{4}{*}{ Actual Classes } & \multicolumn{3}{|c|}{ Predicted Classes } \\
\cline { 2 - 4 } & & 0 (Diabetic) & 1 (Non-Diabetic) \\
\cline { 2 - 4 } & 0 (Diabetic) & 421 & 79 \\
\cline { 2 - 4 } & 1 (Non-Diabetic) & 67 & 201 \\
\hline
\end{tabular}




\section{Comparative Study}

For Validation with diabetes prediction using Rough Set Theory [3]:

Table 5. Comparative Study (validation) with Respect to Rough Set Theory

\begin{tabular}{|l|c|c|c|}
\hline & $\begin{array}{l}\text { Deep Learning } \\
\text { (Type 1 Diabetes } \\
\text { Mellitus) }\end{array}$ & $\begin{array}{l}\text { Deep Learning } \\
\text { (Pima Indians, } \\
\text { Diabetes) }\end{array}$ & $\begin{array}{l}\text { Rough Set } \\
\text { Theory }\end{array}$ \\
\hline $\begin{array}{l}\text { Precision for Non } \\
\text { Diabetic i.e. 0 }\end{array}$ & 0.8064 & 0.75 & 0.7977 \\
\hline $\begin{array}{l}\text { Precision for Diabetic } \\
\text { i.e. 1 }\end{array}$ & 0.7777 & 0.842 & 0.7781 \\
\hline $\begin{array}{l}\text { Recall for Non } \\
\text { Diabetic i.e. 0 }\end{array}$ & 0.9259 & 0.9066 & 0.9133 \\
\hline $\begin{array}{l}\text { Recall for Diabetic i.e. } \\
1\end{array}$ & 0.53846 & 0.55138 & 0.54174 \\
\hline Error Rate & 0.20 & 0.145 & 0.185 \\
\hline
\end{tabular}

\section{Future Work}

This is a rich topic to work on and a lot of further work can be done to improve the efficiency of the neural network in terms of both speed and accuracy. Moreover, using deep learning, AI systems can be created that can predict the onset of diabetes well before a patient is diagnosed with it. Finally, the same model used in this paper can also be applied to a variety of other health problems such as heart diseases, different types of cancers, strokes, respiratory problems and even gall-bladder disease.

\section{Conclusion}

The deep neural network was successfully trained, tested and implemented on the data sets [4][5]. The results obtained were above satisfactory and can be further improved by increasing the size of the data set by adding to it the information gathered by incoming patients in a hospital or in a network of hospitals. Furthermore, the prediction of diabetes can also depend on other factors which are not present in said data set. Taking into consideration these factors will further help to improve the accuracy of the proposed system. Also, the comparison shows that the deep learning models are definitely more effective in terms of precision than the rough set theory model.

\section{References}

[1] A. Shaheen Khan and W. Ahmad, "Intelligent Decision Support System in Diabetic eHealth Care-From the perspective of Elders", Blekinge Institute of Technology, (2009), pp. 88.

[2] T. Saeed Zeki, "An expert system for diabetes diagnosis", American Academic \& Scholarly Research Journal., vol. 4, no. 5, (2012), pp. 1.

[3] R. Ali, J. Hussain, M. Hameed Siddiqi, M. Hussain and S. Lee, "H2RM: A Hybrid Rough Set Reasoning Model for Prediction and Management of Diabetes Mellitus", Sensors, vol. 15, no. 7, (2015), pp. 15921-15951.

[4] A. Frank, "UCI machine learning repository", http://archive. ics. uci. edu/ml, (2010).

[5] National Institute of Diabetes and Digestive and Kidney Diseases, UCI Machine Learning Repository [http://archive.ics.uci.edu/ml]. John Hopkins University, (1990).

[6] T. P. Kamble and S. T. Patil, "Diabetes Detection using Deep Learning Approach", International Journal for Innovative Research in Science \& Technology, vol. 2, no. 12, (2016), pp. 342-349. 
[7] T Chandrakumar and R. Kathirvel, "Classifying Diabetic Retinopathy using Deep Learning Architecture", International Journal of Engineering Research and Technology, vol. 5 no. 06, (2016).

[8] R. Miotto, L. Li, B. A. Kidd and J. T. Dudley, "Deep patient: An unsupervised representation to predict the future of patients from the electronic health records", Scientific reports, vol. 6, (2016).

[9] J. L. Chiang, M. Sue Kirkman, L. M. B. Laffel and A. L. Peters, "Type 1 diabetes through the life span: a position statement of the American Diabetes Association", Diabetes care, vol. 37 no. 7, (2014), pp. 2034-2054.

[10] V. Gulshan, L. Peng, M. Coram, M. C. Stumpe, D. Wu, A. Narayanaswamy, S. Venugopalan, K. Widner, T. Madams, J. Cuadros and R. Kim, "Development and validation of a deep learning algorithm for detection of diabetic retinopathy in retinal fundus photographs", JAMA, vol. 316, no. 22, (2016), pp. 2402-2410.

[11] S. Choi, E. Kim and S. Oh, "Human behavior prediction for smart homes using deep learning", ROMAN, IEEE, (2013), pp. 173-179.

[12] M. Anouncia S, C. Madonna L. J., P. Jeevitha and R. T. Nandhini, "Design of a Diabetic Diagnosis System Using Rough Sets", Cybernetics and Information Technologies, vol. 13, no. 3, (2013), pp. 124139.

[13] R. Zolfaghari, "Diagnosis of Diabetes in Female Population of Pima Indian Heritage with Ensemble of BP Neural Network and SVM", International Journal of Computational Engineering \& Management, vol. 15, no. 4, (2012), pp. 2230-7893.

[14] K. Sridar and D. Shanthi, "Medical diagnosis system for the diabetes Mellitus by using back propagation-AprioriAlgorithms", Journal of Theoretical and Applied Information Technology, vol. 68, no. 1, (2014).

[15] G. Kaur, "Improved J48 Classification Algorithm for the Prediction of Diabetes", International Journal of Computer Applications, vol. 98, no. 22, (2014).

[16] G. van Tulder and M. de Bruijne, "Learning Features for Tissue Classification with the Classification Restricted Boltzmann Machine", springer international publishing, (2014), pp. 47-58.

[17] A. Fischer and C. Igel, "An Introduction to Restricted Boltzmann Machines", Springer, (2012), pp. 1436.

[18] A. Fischer, Thesis, “Training Restricted boltzman machine”, Université de Montréal, (2006).

[19] I. Pole, "Restricted Boltzmann Machine - A comprehensive study with a focus on Deep Belief Networks", Student developer at Daenet GmbH.

[20] D. Mo, "A survey on deep learning: one small step toward AI", University of New Mexico, USA, (2012).

[21] W. Smith Jack, J. E. Everhart, W. C. Dickson, W. C. Knowler and R. S. Johannes, "Using the ADAP learning algorithm to forecast the onset of diabetes mellitus", Proceedings of the Annual Symposium on Computer Application in Medical Care, American Medical Informatics Association, (1988), November 9.

[22] J. Lamb, "MATH 3220 Final Project".

[23] Y. Bengio, "Learning Deep Architectures for AI", Foundations and trends® in Machine Learning, vol. 2, no. 1, (2009), pp.1-127.

[24] L. Deng and D. Yu, "Deep Learning Methods and Applications”, Foundations and Trends ${ }^{\circledR}$ in Signal Processing, vol. 7, no. 3-4, (2014), pp. 197-387.

[25] "Rosenblatt's Perceptron", chapter 1.

[26] M. ArifWani Saduf, "Comparative Study of Back Propagation Learning Algorithms for Neural Networks", International Journal of Advanced Research in Computer Science and Software Engineering, vol. 3, no. 12, (2013), pp. 1151-1156.

[27] M. A. Wiering, M. Schutten, A. Millea, A. Meijster and L. R. B. Schomaker, "Deep Support Vector Machines for Regression Problems", Institute of Artificial Intelligence and Cognitive Engineering, (2013).

[28] Y. Kumar Jain and S. Kumar Bhandare, "Min Max Normalization Based Data Perturbation Method for Privacy Protection", International Journal of Computer \& Communication Technology, vol. 2, no. 8, (2011), pp. 45-50.

[29] J. M. Tomczak, "Application of Classification Restricted Boltzmann Machine with discriminative and sparse learning to medical domains", Institute of Computer Science Wroclaw University of Technology. 


\section{Authors}

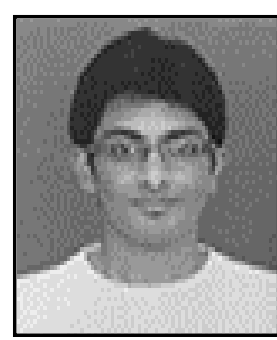

Sushant Ramesh, passed out student, of Bachelors of Technology in Computer Science and Engineering at VIT University, Vellore, Tamil Nadu, India. He has high enthusiasm and ambition towards the projects he works on. His academic interests include Artificial Intelligence (primarily Fuzzy Logic and Artificial Neural Networks) and Embedded Systems.

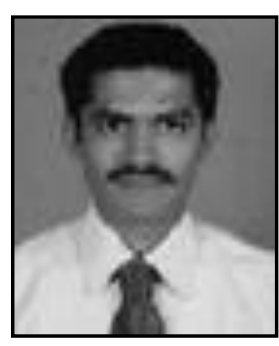

H. Bajaj currently working as an Associate Professor in Computer Science and Engineering Department at Sreenidhi Institute of Science and Technology, Hyderabad, Telangana India. His areas of research include Data Warehousing and Mining and Network Security.

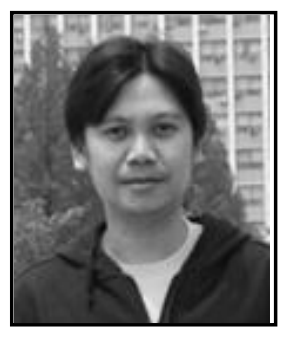

Ronnie D. Caytiles, he had his Bachelor of Science in Computer Engineering- Western Institute of Technology, Iloilo City, Philippines, and Master of Science in Computer Science- Central Philippine University, Iloilo City, Philippines. He finished his Ph.D. in Multimedia Engineering, Hannam University, Daejeon, Korea. Currently, he serves as an Assistant Professor at Multimedia Engineering department, Hannam University, Daejeon, Korea. His research interests include Mobile Computing, Multimedia Communication, Information Technology Security, Ubiquitous Computing, Control and Automation

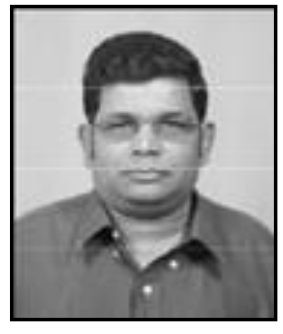

N. Ch. S. N. Iyengar (b 1961), he currently Professor at the Sreenidhi Institute of Science and Technology (SNIST) Yamnapet, Ghatkesr, Hyderabad, Telengana, India. His research interests include Agent-Based Distributed Computing, Intelligent Computing, Network Security, Secured Cloud Computing and Fluid Mechanics. $\mathrm{He}$ had 32+ years of experience in teaching and research, guided many scholars, has authored several textbooks and had nearly 200+ research publications in reputed peer reviewed international journals. He served as PCM/reviewer/keynote speaker/ Invited speaker. 
International Journal of Database Theory and Application Vol.10, No.9 (2017) 